\title{
Clinician-led improvement in cancer care (CLICC) - testing a multifaceted implementation strategy to increase evidence-based prostate cancer care: phased randomised controlled trial - study protocol
}

Bernadette (Bea) Brown ${ }^{1,2^{*}}$, Jane Young ${ }^{2}$, David P Smith ${ }^{3,4}$, Andrew B Kneebone ${ }^{5,6}$, Andrew J Brooks ${ }^{7,8,9}$, Miranda Xhilaga ${ }^{10}$, Amanda Dominello ${ }^{1}$, Dianne L O'Connell ${ }^{2,3,11,12}$ and Mary Haines ${ }^{1,2}$

\begin{abstract}
Background: Clinical practice guidelines have been widely developed and disseminated with the aim of improving healthcare processes and patient outcomes but the uptake of evidence-based practice remains haphazard. There is a need to develop effective implementation methods to achieve large-scale adoption of proven innovations and recommended care. Clinical networks are increasingly being viewed as a vehicle through which evidence-based care can be embedded into healthcare systems using a collegial approach to agree on and implement a range of strategies within hospitals. In Australia, the provision of evidence-based care for men with prostate cancer has been identified as a high priority. Clinical audits have shown that fewer than 10\% of patients in New South Wales (NSW) Australia at high risk of recurrence after radical prostatectomy receive guideline recommended radiation treatment following surgery. This trial will test a clinical network-based intervention to improve uptake of guideline recommended care for men with high-risk prostate cancer.
\end{abstract}

Methods/Design: In Phase I, a phased randomised cluster trial will test a multifaceted intervention that harnesses the NSW Agency for Clinical Innovation (ACI) Urology Clinical Network to increase evidence-based care for men with high-risk prostate cancer following surgery. The intervention will be introduced in nine NSW hospitals over 10 months using a stepped wedge design. Outcome data (referral to radiation oncology for discussion of adjuvant radiotherapy in line with guideline recommended care or referral to a clinical trial of adjuvant versus salvage radiotherapy) will be collected through review of patient medical records. In Phase II, mixed methods will be used to identify mechanisms of provider and organisational change. Clinicians' knowledge and attitudes will be assessed through surveys. Process outcome measures will be assessed through document review. Semi-structured interviews will be conducted to elucidate mechanisms of change.

Discussion: The study will be one of the first randomised controlled trials to test the effectiveness of clinical networks to lead changes in clinical practice in hospitals treating patients with high-risk cancer. It will additionally provide direction regarding implementation strategies that can be effectively employed to encourage widespread adoption of clinical practice guidelines.

Trial registration: Australian New Zealand Clinical Trials Registry (ANZCTR): ACTRN12611001251910.

Keywords: Implementation strategies, Clinical practice guidelines, Clinical networks, Cancer, Interventions

\footnotetext{
* Correspondence: bea.brown@saxinstitute.org.au

${ }^{1}$ Sax Institute, Haymarket, Australia

${ }^{2}$ School of Public Health, University of Sydney, Camperdown, Australia

Full list of author information is available at the end of the article
} 


\section{Background}

\section{The evidence-practice gap}

The discrepancy between research evidence and clinical practice is well documented [1], and remains one of the most persistent problems in providing high-quality healthcare [2]. Clinical practice guidelines have been extensively developed as a means to disseminate best practice and ensure clinical decision-making is informed by recent, credible research evidence, thereby improving healthcare processes and outcomes. However, timely and effective implementation of guidelines into clinical practice is inconsistent [3], and it remains surprisingly difficult to make changes across the health system even when there is compelling evidence [4].

The difficulty in achieving large scale adoption of proven innovations and recommended care (as well as discontinuing ineffective or harmful practices) has been characterised as a 'translation block' [5-8].

\section{Effective implementation}

Previous research indicates that successful implementation of evidence-based care depends critically on the extent to which strategies address prospectively identified barriers, through theoretical frameworks of behaviour change [9,10], and promote provider acceptance [3]. Recommendations from clinical guidelines are more likely to become embedded within practice when they: are initiated and led by local clinical leaders; are tailored to the local context; and engage clinicians in the design of the implementation strategy [1,3,11-13]. Grol [14] argues that to effectively implement evidence-based practice, research urgently has to change so that it develops through collaborations between clinicians, researchers, patients, policy makers, and quality improvement experts.

Specifically, the growing body of evidence suggests several core implementation strategies are effective in bringing about system-wide and sustained change $[1,11,15,16]$ :

1. Clinical champions/leaders supporting change within their practices and settings;

2. System, structural, and organisational support for system-wide changes to enable implementation strategies to be rolled out and scaled up (e.g., legislation, resources, mechanisms for communication and collaboration between health sectors);

3. Ongoing monitoring, evaluation, and feedback of changes as they are implemented.

\section{Clinical networks - a medium for implementation}

In New South Wales (NSW), Australia, a coordinated program of 30 clinical networks, institutes and taskforces has been established by the NSW Agency for Clinical Innovation (ACI), a board-governed statutory organisation funded by the NSW Ministry of Health.
These clinical networks of volunteer health professionals provide a framework for doctors, nurses, allied health professionals, managers, and consumers to collaborate across regional and service boundaries to drive improvements in service delivery and care outcomes through innovation in clinical practice.

This type of non-mandatory clinical network is increasingly being viewed as a vehicle through which evidencebased care can be embedded into healthcare systems using a collegial approach to agree on and implement a range of strategies within hospitals. They provide 'bottom up' views on the best ways of tackling complex healthcare problems coupled with the strategic and operational 'top down' support necessary to facilitate and champion changes in practice at the clinical interface $[17,18]$. There is evidence from 'before and after' controlled studies that when clinical practice guidelines are implemented through clinical networks there are improvements in compliance with guideline recommendations and the quality of care $[19,20]$.

Clinical networks embody, or have the potential to enable, the core features of successful implementation strategies and therefore are a mechanism for health system change and increasing the uptake of evidence-based care for three reasons:

1. Clinical networks contain clinical leaders who can design and champion change to improve care within their practices and influence wider culture change within their healthcare settings.

2. Clinical networks are a 'ready-made' organisational structure through which innovations may be promulgated and accelerated by clinicians.

3. Clinical networks provide a structure to monitor and evaluate changes as they are implemented to answer questions about effectiveness and the success of implementation strategies.

\section{Prostate cancer clinical practice guidelines-an opportunity} to translate research into effective healthcare practice

Prostate cancer is the most common cancer registered in Australia and is the second highest cause of cancer death in males [21]. Radical prostatectomy is the most frequent procedure for localised prostate cancer, however following surgery it is estimated that $20 \%$ to $50 \%$ of men are at 'high risk' of experiencing progression or recurrence [22-25]. A national strategy to improve prostate cancer services and thereby improve patients' quality of life and survival identified the provision of evidencebased care for these men as a high priority [26]. Persuasive evidence from randomised controlled trials indicates the need to alter current practice by offering radiotherapy to men with adverse disease features following surgery as radiotherapy treatment halves the risk of 
recurrence [27-29] and improves biochemical diseasefree survival [30]. A Grade B recommendation (denoting that the Clinical Practice Guideline expert working group considered that the body of evidence can be trusted to guide practice in most situations) in the Clinical Practice Guidelines for the Management of Locally Advanced and Metastatic Prostate Cancer produced by the Australian Cancer Network [31] recommends that 'patients with extracapsular extension, seminal vesicle involvement or positive surgical margins receive postoperative external beam radiation therapy within four months of surgery.' This recommendation is echoed in the more recently published American Urological Association Guideline, Adjuvant and Salvage Radiotherapy after Prostatectomy, which states 'Physicians should offer adjuvant radiotherapy to patients with adverse pathologic findings at prostatectomy (Standard; Evidence Strength: Grade A)' [32]. The most recently available data indicate less than $10 \%$ of patients with locally advanced prostate cancer in NSW Australia receive guideline recommended care [33]. Patterns of care for prostate cancer in NSW generally reflect practice in other Australian jurisdictions [34,35]. These data are consistent with that from the United States where less than $20 \%$ of eligible patients receive adjuvant radiotherapy, indicating substantial room for improvement [36]. Current evidence about strategies to encourage the adoption of clinical practice guidelines is limited [1-3,9,37] and provides little clear direction about approaches that can be effectively employed in specific settings.

\section{Aims}

The aim of this study is to develop and trial a locally tailored, multifaceted implementation strategy that harnesses the NSW Agency for Clinical Innovation (ACI) Urology Clinical Network to increase evidence-based care for men with high-risk prostate cancer following prostatectomy in selected NSW hospitals [31]. Specifically, the aim is to increase referral to radiation oncology for a discussion about radiotherapy, and the associated risks and benefits of treatment, to support fully informed decision making.

An additional aim is to identify reasons why changes in behaviour and outcomes occurred or did not occur in study hospitals and why the implementation strategy did or did not result in increased compliance with guideline recommended care.

If the intervention is successful we will also assess the sustainability of increases in referral patterns within the hospitals through interviews with key stakeholders.

\section{Approach to intervention design}

Any reason for resisting new practice is a barrier to change and the potential importance of such barriers and their influence on quality improvement activities has been highlighted in numerous studies [38-41]. A recent systematic review indicates that tailored interventions are more effective when they are designed to address prospectively identified local barriers to change [10]. A key component of our method is to tailor our intervention so that it incorporates features that will facilitate changes in provider behaviour by addressing local level obstacles.

Intervention elements have been informed by reviews of the clinical practice change literature [9,11,37,38,42-61], and refined and tailored to take account of the organisational context in which providers practice through a multi-component needs and barriers analysis, including: iterative workshops with members of the ACI Urology Clinical Network; a national baseline survey (offered in web-based and paper form) of all urologist members of the Urological Society of Australia and New Zealand, the peak professional body, to explore current knowledge, attitudes and practice in the wider context (results published elsewhere); semi-structured interviews with urology, radiation oncology, and nursing staff at target hospitals to explore site specific practice and barriers; consumer feedback on what information patients want from their urologist; and consultation with a cancer policy advisory group to ensure intervention elements are feasible, scalable and potentially translatable to other cancers (see Figure 1 for summary).

Results from these activities indicate that, in priority order, barriers can be grouped into three main clusters:

1. Clinician: attitudes and beliefs held by individual clinicians about the validity of the evidence base supporting the guideline recommendation (54\% of urologists surveyed agreed that the recommendation is based on a valid interpretation of the underlying evidence) - notably due to ongoing clinical trials, which raise doubts as to the treatment benefit of adjuvant radiotherapy versus early salvage radiotherapy; concerns about overtreatment and toxicity/side effects associated with radiotherapy and lack of familiarity with current radiotherapy techniques (two thirds of urologists surveyed agreed that patients may experience unnecessary discomfort if they follow the recommendation).

2. Patient: treatment preferences (perceived to be influenced by interaction with urologists).

3. Hospital system and processes: variation in urologists' engagement with the multidisciplinary team (MDT) of specialist surgeons, medical oncologists, radiation oncologists, nurses and other allied health professionals providing specialist cancer care; and selective presentation of high-risk prostate cancer cases to the MDT resulting in inconsistent 


\section{Literature review}

Components of interventions that have been successfully used in the implementation of clinical practice guidelines

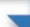

Iterative workshops

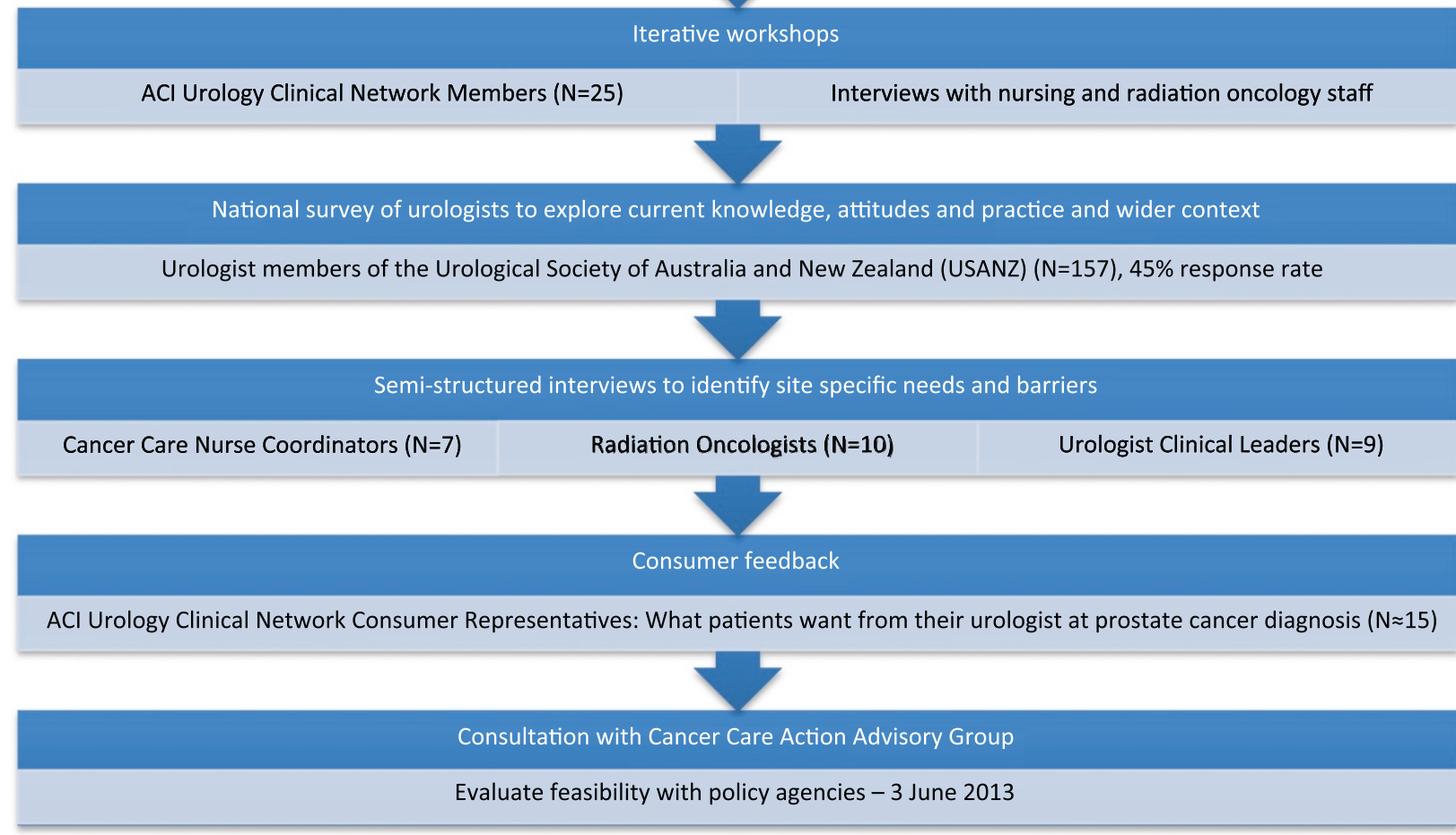

Figure 1 Approach to intervention design.

multidisciplinary discussion of all available treatment options and pathways.

\section{Conceptual model}

Intervention components are underpinned by the PRECEDE-PROCEED theory of behaviour change [62,63] that relates interpersonal factors and system characteristics into one model to inform change in practice. This theory enables the integration of barriers identified through our mixed methods needs and barriers analysis into 'predisposing factors' (e.g., knowledge and attitudes of the target group); 'reinforcing factors' (e.g., opinions and behaviour of peers); and 'enabling factors' (e.g., capacity of the system and hospital processes). This is one of the most widely used theories to support rigorous trials of the implementation of guidelines [16] and systematic reviews have shown that trials that intervene to alter these three factors are the most successful [13]. Figure 2 illustrates how the identified barriers to change in prostate cancer care have been grouped into the factors of the PRECEDEPROCEED theory. Additionally, Figure 2 illustrates the intervention components that have been designed to target each barrier.

\section{Intervention components}

Physician-focused components

1. Provider education (predisposing factor): The Urologist Clinical Leader at each hospital will be supported to facilitate an interactive education session at a routinely scheduled multidisciplinary team (MDT) meeting. This session will be moderated by members of the research team to ensure fidelity and will last approximately 10 to 15 minutes. Participants will be presented with an introduction to the study, including a summary of the evidence underlying the guideline recommendation through a video presentation to control for inconsistency across sites. The video includes the Co-Chair of the ACI Urology Clinical Network, a peer-identified national urologist opinion leader, and a consumer who introduce key messages through discussion of their practice and experience.

2. Dissemination of printed materials (predisposing factor): In the active implementation phase all urologists will be given a full copy of the Clinical Practice Guidelines for the Management of Locally 


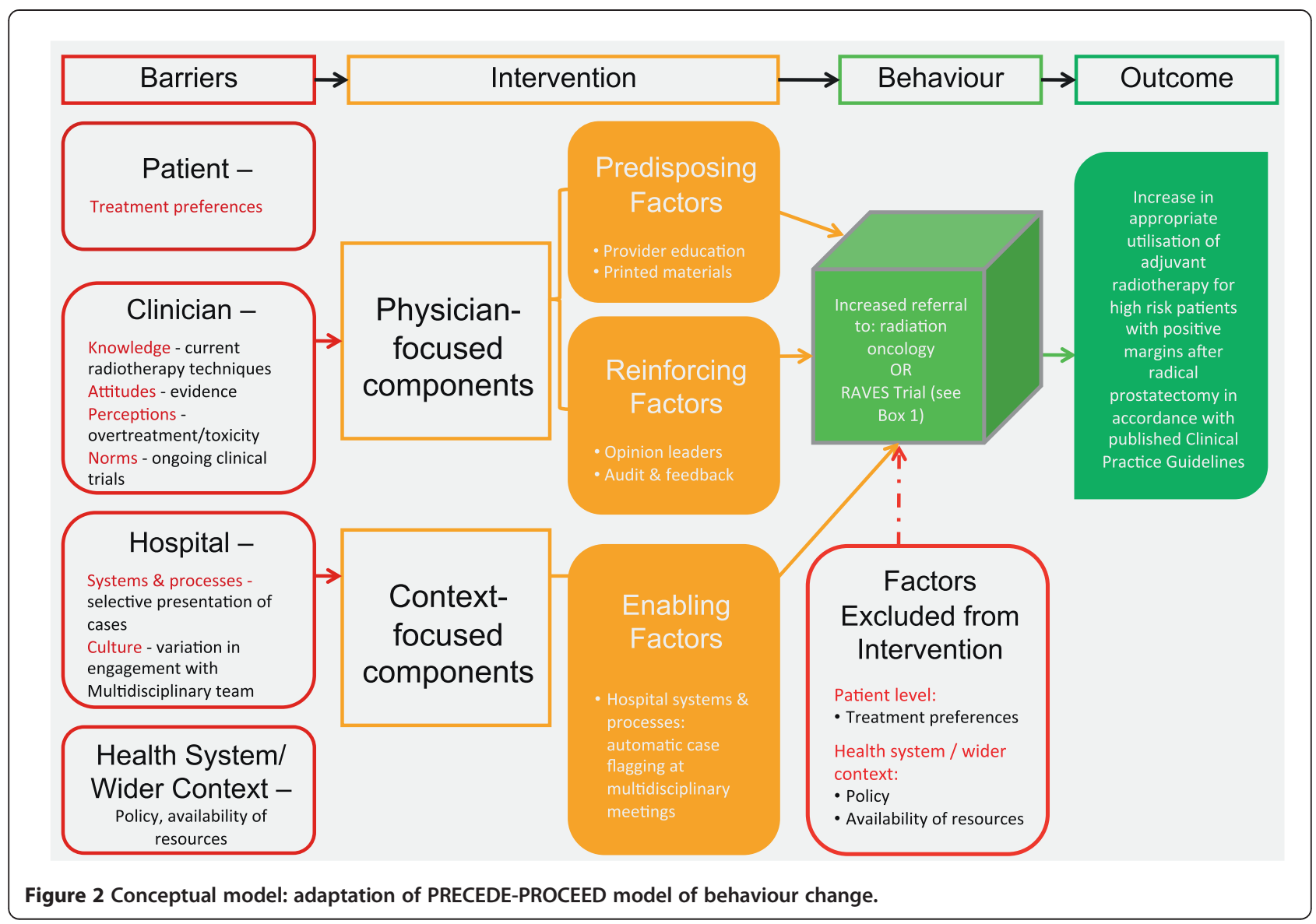

Advanced and Metastatic Prostate Cancer and a summary card that allows quick reference to the evidence supporting the specific recommendation that is the focus of the study, together with information on potential side effects and toxicity. The reverse of this summary card provides information on current radiotherapy techniques and key points to guide impartial discussion with patients before and after surgery to support fully informed decision-making. This includes the potential need for multidisciplinary care and consultation with a radiation oncologist to obtain information about what radiotherapy would involve and the likely benefits and risks of treatment if high-risk features are found upon histopathological examination of the prostate specimen.

3. Opinion leaders (reinforcing factor): A key aspect of the intervention will be the use of Urologist Clinical Leaders in each hospital, identified by peers as being educationally influential, to engage the target group. Clinical Leaders will reinforce key messages, persuade peers to participate in the study and will model targeted referral behaviours and promote practice change [64]. Following the education session, Clinical Leaders will provide ongoing peer support and engage in discussions with colleagues to seek and provide feedback on practice and any continuing barriers to change. The Clinical Leaders are members of the ACI Urology Clinical Network and were recruited by the Network Co-Chair, an expert opinion leader who is influential due to his authority and status amongst his peers [65]. The introduction of key messages by a national opinion leader in the video presented at the education session provides an additional level of peer-to-peer influence.

4. Audit and feedback (reinforcing factor): Following commencement of the intervention, urologists will be provided with ongoing feedback reports detailing the number of patients referred to radiation oncology, at the individual, hospital and study level, obtained through data extraction from medical records. The feedback report will also include information on the number of patients at high risk who are discussed at MDT meetings. The initial feedback report will include baseline data. Feedback will be provided via email or SMS depending on the preferred method of communication of each participant. Aggregated quarterly feedback reports 
will additionally be presented verbally by the Clinical Leader at MDT meetings.

\section{Context-focused components}

Guideline dissemination and educational components will address gaps in provider knowledge. However, a number of reviews indicate that increased knowledge is necessary but insufficient to change individual or organisational behaviour [41]. It is also necessary to enable change by increasing means or reducing barriers [66]. Therefore, in conjunction with physician-focused components, utilising the leverage of the ACI Urology Clinical Network to address the systems barriers identified through the mixed methods needs and barriers analysis, context-focused components will include a new system for automatic case flagging at MDT meetings (enabling factor). Urologists practising at the nine target hospitals will be requested to provide consent for the names of all patients who have had a histopathological examination of a radical prostatectomy specimen and who have extracapsular extension, positive surgical margins or seminal vesicle involvement to be submitted automatically to the hospital urology MDT meeting for discussion. Pathology providers will provide a list of all eligible patients to the MDT coordinator. This will reduce variation in practice and selective presentation of cases to the MDT meeting with the intent to promote more collaborative decision-making and increased referral to radiation oncology for high-risk patients.

\section{Methods}

Phase I: intervention rollout and implementation trial Hypotheses

Compared with pre-intervention, a larger proportion of post-operative radical prostatectomy patients who are at high risk of recurrence (have extracapsular extension, seminal vesicle involvement or positive surgical margins) treated in hospitals after implementation of the intervention will receive a referral to radiation oncology for consideration of adjuvant radiotherapy or referral to the RAVES trial [Radiotherapy Adjuvant Vs Early Salvage (Protocol Number: TROG.08.03); see the 'RAVES Trial' subsection for details].

\section{Design}

This will be a phased randomised cluster trial with phased introduction of a clinical network led organisational intervention in nine hospitals over 10 months. The order in which hospitals will receive the intervention will be determined randomly using a stepped wedge study design (see Figure 3). This design, originally developed for community studies, has more recently been applied to health service interventions in hospitals [67] and has the following advantages: provides a control comparison where geographic controls are not possible; allows all hospitals in the clinical network with multidisciplinary teams to take part in the intervention; enables the intervention to be tested within the parameters of real-world allocation of clinical network resources with a phased roll out of the hospital-based intervention; and complies with the Cochrane Effective Practice and Organisation of Care Group's consensus statement about study designs of sufficient quality to be included in systematic reviews. This study will be conducted and reported in accordance with the CONSORT statement for the reporting of pragmatic trials $[68,69]$.

The intervention will be rolled out across the nine hospitals in five steps of two-month blocks from December 2013 to September 2014. Throughout the study, hospitals will either be in the active implementation

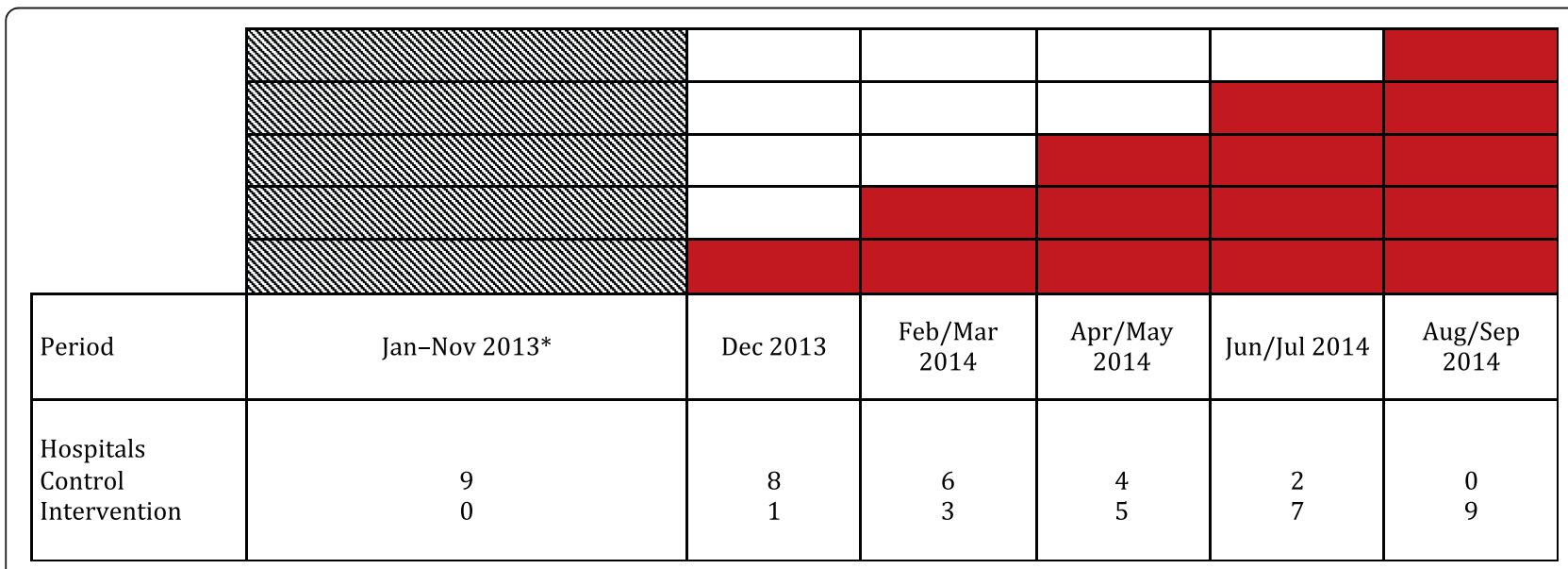

Figure 3 Stepped wedge study design: staged rollout of intervention from December 2013 to September 2014. The solid shaded blocks represent introduction of the intervention over 5 steps. The intervention will be rolled out across the nine hospitals in two-month blocks. Patient medical records will be reviewed for a period of 12 months following the interactive education session. Therefore data collection will not be completed until September 2015. *Control-only monitoring not part of the intervention study. 
(intervention) or passive (control) phase (see Figure 3). Eligibility criteria for inclusion are public hospitals: with a urology multidisciplinary team (MDT) comprising specialists, nurses, and allied health professionals; and that are members of the ACI Urology Clinical Network and have a urologist who will act as the Clinical Leader for that site. All urologists who are members of the urology multidisciplinary team at intervention hospitals will be eligible for inclusion ( $\mathrm{n} \approx 4-10$ urologists per hospital).

\section{Outcomes}

Primary outcomes are patient referral to radiation oncology for discussion of adjuvant radiotherapy in line with guideline recommended care or referral to the RAVES trial (see the 'RAVES Trial' subsection for details). Secondary outcomes include: an initial patient consultation with a radiation oncologist; enrolment in the RAVES trial; and commencement of radiotherapy.

\section{RAVES Trial - an opportunity to demonstrate shift in equipoise}

RAVES [Radiotherapy Adjuvant Vs Early Salvage (Protocol Number: TROG.08.03)] is a multi-centre phase III clinical trial comparing survival and quality of life outcomes for patients at high-risk post prostatectomy who are randomised to have: i) radiotherapy deferred (salvage radiotherapy) until their prostate specific antigen (PSA) begins to rise (common current practice); OR ii) immediate radiotherapy (adjuvant radiotherapy) after surgery (regarded as evidence-based standard of care). This is seen as a very important local trial as, despite international evidence that adjuvant radiotherapy is effective, this practice has not been widely adopted due to Urologists' concerns about side effects and overtreatment. The aim of the RAVES trial is to determine whether salvage radiotherapy is as effective as adjuvant radiotherapy and results in improved quality of life.

\section{Data collection-data extraction from patients' medical records}

Outcome data to assess changes in healthcare practice will be collected through data extraction from urologists' and radiotherapy patients' medical records by independent, trained research assistants who are blind to the date that the intervention was commenced at the hospital. Baseline data will be collected retrospectively for patients undergoing a radical prostatectomy during January 2013 to November 2013. Pilot testing of the medical record review tools and processes will allow us to train the research assistants and establish and test data collection procedures.

\section{Information from medical records}

Treatment outcomes that will be collected through medical record review for cases with extracapsular extension, seminal vesicle involvement or positive surgical margins (confirmed by pathology reports) are: referral to radiotherapy, taken from the surgeon's notes (including dates of surgery and referral) or in the case where there was no referral that radiotherapy was discussed and the reason(s) for not referring to radiotherapy; uptake of radiotherapy or enrolment into the RAVES trial from the radiation oncology database; and time between surgery and commencement of radiotherapy. Individual case records will be reviewed for a minimum of six months after initial radical prostatectomy.

Data will be abstracted from medical records at hospitals, cancer centres and urologists' private consulting rooms using previously established methods [33].

Hospital level factors will be collected from centrally held records including specialist cancer centre and size. Patient level factors will be collected from the medical and hospital records including: month and year of birth, comorbidities, stage of cancer, Gleason score, PSA level at diagnosis, country of birth and private health insurance status. Remoteness of residence and socio-economic status (SES) of the cases will be assigned using their postcode of residence and the ARIA [70] and SEIFA [71], respectively.

Hormone therapy, comorbidities, pre-diagnostic PSA levels, Gleason score, country of birth, and health insurance status are potential barriers to referral for radiotherapy.

\section{Study sample}

The unit of study will be the participating multidisciplinary teams (MDT). Nine public hospital-based MDTs in NSW will participate. The hospitals are located in both metropolitan and regional areas. Approximately four to ten urologists will be included at each site.

\section{Data analysis}

The primary analysis will be conducted at the individual patient level using a generalized estimating equations (GEE) approach to account for repeated outcome observations within clusters (urologists and MDTs). The dependent variable for this analysis will be referral to a radiation oncology service for adjuvant radiotherapy or enrolment into the RAVES trial (versus no referral) for each prostate cancer case. The exposure variable will be the intervention status (pre versus post) of the hospital at the time of the post-prostatectomy consultation. Other independent variables will be added to the model if they are shown to be independently associated with radiotherapy referral and/or their inclusion in the model changes the linear coefficient of the intervention effect by more than $20 \%$ in absolute value. Analysis to determine the extent to which changes in urologists' knowledge, attitudes and beliefs (Phase II) mediated any changes in referral patterns will be assessed by including clinicians' change scores in the GEEs. 


\section{Sample size and statistical power}

Based on estimates from the NSW Central Cancer Registry and Medicare claims data we estimate that 3,517 NSW men will have a radical prostatectomy in 2013. Approximately 1,618 (46\%) of these will be performed in the nine hospitals with urological MDTs participating in the ACI Urology Clinical Network according to linked cancer registry and hospital data for all NSW men diagnosed with prostate cancer. Assuming no major change has occurred in this distribution, there will be 1,348 radical prostatectomies over the 10 months of this trial. Of these, 20 to $50 \%$ or 270 to 671 men will be at 'high risk' [22-25]. The stepped wedge design is relatively insensitive to variations in the intracluster correlation (ICC) as a consequence of its efficient use of within-cluster and between-cluster information and has little impact on the study's power. However, based on the best available information, we estimate that the ICC for use of radiotherapy will be between 0.09 and 0.15 [72].

The most recently available data indicate $10 \%$ of highrisk men receive radiotherapy after surgery in NSW [33]. With the release of the Australian Cancer Network Clinical Practice Guidelines and the commencement of the RAVES trial we estimate that at the commencement of our trial, administration of radiotherapy following surgery will have increased to $15 \%$ to $20 \%$ of high-risk patients. Our stepped wedge study design with nine clusters, six time intervals (including the pre-intervention control step) and ICCs of 0.09 to 0.15 will have at least $80 \%$ power to detect an increase in referral to a radiation oncologist from $15 \%$ to $35 \%$, or $20 \%$ to $40 \%$ if a minimum of $30 \%$ of patients are at high risk, and from $20 \%$ to $35 \%$ if at least $50 \%$ of prostate cancer cases are at high risk.

\section{Staff training and evaluation}

Primary and secondary outcomes can be measured reliably through clinical data collection and this method has been used previously $[33,73,74]$. Research assistants conducting the medical record review will be trained and we will conduct a $10 \%$ blinded re-review to assess inter-rater reliability.

\section{Phase II: identify mechanisms of provider and organisational change Design}

'Before and after' mixed methods study to measure knowledge, attitudes, process, and explanatory variables.

\section{Urologists' knowledge and attitudinal outcomes Hypotheses}

Compared with pre-intervention measures, urologists post-intervention will have: increased knowledge about the evidence for appropriate adjuvant radiotherapy for high-risk prostate cancer patients after radical prostatectomy and the associated risks and benefits of treatment; and more positive attitudes towards the need for referral to radiation oncology as a means to support fullyinformed patient decision making.

\section{Data collection}

A quantitative study of urologists will be conducted using a questionnaire to assess knowledge, beliefs, social influences, attitudes and motivation at three time points: baseline (pre-intervention); six months after the roll-out of the intervention; and at the end of the study $(n \approx 4-$ 10 urologists per hospital). The survey is tailored to the intervention, uses previously identified domains (knowledge, beliefs, motivation, social influences), constructs, and generic questions to investigate the implementation of evidence-based practice [48], and is modelled on questions developed for other clinical conditions [75]. The measures using Likert scales have been developed through pilot testing and their feasibility and reliability will be assessed as part of the data collection in accordance with best practice [76]. Questions are consistent with those used in the baseline nationwide survey of urologists to enable comparison between groups. These surveys produce continuous scores for knowledge, beliefs, social influences, attitudes, and motivation at the clinician level that will be averaged for each hospital at each time point.

A follow up nationwide survey of urologist members of the Urological Society of Australia and New Zealand (USANZ) $(n \approx 370)$ will be conducted to determine whether urologists' attitudes shifted locally/nationally without intervention.

\section{Process outcomes}

\section{Research question}

Was the intervention implemented as intended?

\section{Data collection}

The date of commencement of the intervention will be noted as the day the Urologist Clinical Leader within each site facilitated the educational intervention session. Agendas and minutes of subsequent MDT meetings will be reviewed using a method developed by members of the investigator team [77] to assess: numbers attending the meeting; frequency of mentioning the study; discussion of cases flagged by pathology; presentation of medical record review feedback; and changes in hospital practice as indicators of sustained interest in the intervention and organisational process changes.

\section{Research questions}

1. Why did or did not the intervention result in evidence-based care?

2. Why was or was not the intervention implemented or sustained in hospitals? 


\section{Data collection}

1. Qualitative semi-structured interviews with Clinical Leaders at the end of the study to feedback study results and explore the reasons for them $(n=9)$.

2. Qualitative semi-structured telephone interviews, informed by feedback from Clinical Leaders, with urologists in the nine intervention hospitals at the end of the study to feedback study results and further explore the reasons for them $(n \approx 4-10$ urologists per hospital).

\section{Data analysis}

Survey data will be analysed using bivariable methods (means, t-tests and ANOVA for normally distributed continuous data; medians and non-parametric tests for non-normally distributed continuous data; and proportions and chi-squared tests for categorical data).

Semi-structured interview data will be analysed thematically using a matrix-based framework to organise data according to the theoretical framework used for the intervention design to identify why changes did or did not happen in the hospitals and why the intervention did or did not result in improved care.

\section{Research governance}

The study has been approved by Royal Prince Alfred Research Ethics Committee (ID: X12-0388 \& HREC/12/ $\mathrm{RPAH} / 584$ ). Site-specific approval (SSAs) from the research governance office at each of the nine participating hospitals has been obtained. Site-specific approval from Cancer Council NSW ethics committee has been granted to cover data collection, storage and analysis at Cancer Council NSW.

\section{Trial status}

The intervention and data collection phase of the study commenced in December 2013.

\section{Discussion}

Clinical networks such as those established by the NSW Agency for Clinical Innovation are increasingly being viewed as an important strategy for increasing evidencebased practice in Australia and other countries. This interest in clinical networks is accompanied by significant investment in them but few studies have directly tested their effectiveness in driving implementation initiatives. To the authors' knowledge, this study will be one of the first randomised controlled trials to test the effectiveness of clinical networks to lead changes in clinical practice in hospitals treating patients with high-risk cancer and improve evidence-based care.

\section{Limitations}

The aim of this study is to target referral patterns of practising clinicians using the leverage of a clinical network. Intervention components therefore focus on the attitudinal and systems barriers at the urologist and hospital level. While we have sought consumer input into the design of provider-focused materials to provide guidance on what information patients want from consultation with their physician, ethics approval for the current study does not permit direct interaction with patients being treated by urologists in the study. The research team is developing a proposal for a sub-study focused on how patients can influence the treatment they receive, to be conducted at the end of Phase I.

\begin{abstract}
Abbreviations
ACl: NSW agency for clinical innovation; ARIA: Accessibility/remoteness index of Australia; Cl: Chief investigator; CLICC: Clinician-led improvement in cancer care; GEE: Generalised estimating equation; HREC: Human research ethics committee; ICC: Intracluster correlation; MDT: Multidisciplinary team; MRN: Medical record number; NHMRC: National health and medical research council; NSW: New South Wales; PSA: Prostate specific antigen; RAVES: Radiotherapy adjuvant vs early salvage (phase iii randomised trial); SEIFA: Socio-economic indexes for areas; SES: Socioeconomic status; SSA: Site-specific approval; USANZ: Urological Society of Australia and New Zealand.
\end{abstract}

\section{Competing interests}

Miranda Xhilaga is employed by the Prostate Cancer Foundation of Australia (PCFA), which has provided funds to support this research as part of the National Health and Medical Research Council (NHMRC) of Australia's partnership project grant scheme (ID: 1011474). Andrew Brooks is the CoChair of the NSW Agency for Clinical Innovation (ACI) Urology Clinical Network and Mary Haines is on the Research Sub-Committee of the Agency for Clinical Innovation Board. This Agency has provided in-kind funds as part of the National Health and Medical Research Council (NHMRC) of Australia's partnership project grant scheme (ID: 1011474). The contents of this paper are solely the responsibility of the individual authors and do not reflect the views of the National Health and Medical Research Council of Australia, Prostate Cancer Foundation of Australia or NSW Agency for Clinical Innovation. The other authors declare that they have no competing interests.

\section{Author's contributions}

The authors are the chief, associate, and honorary investigators of the research grant funding this research activity. BB, in collaboration with all other authors, conceptualised the research project and developed the protocol presented in this paper. All authors provided input into various aspects of the study, provided ongoing critique, and approved the final version of the manuscript.

\section{Acknowledgements}

As well as the named authors, study contributors are: Cyra Patel (Sax Institute), Jane Bois (Sax Institute), Sam Egger (Cancer Council NSW), Kristie Weir (Cancer Council NSW), Maria Albania (Cancer Council NSW), Leighna Carmichael (Cancer Council NSW), Violeta Sutherland (NSW Agency for Clinical Innovation), Donald McLellan (NSW Agency for Clinical Innovation). This research is supported by the National Health and Medical Research Council (NHMRC) of Australia through their partnership project scheme (ID: 1011474). The Prostate Cancer Foundation of Australia also provided funds to support this research as part of the NHMRC partnership project grant scheme.

This study is registered with the Australian New Zealand Clinical Trials Registry (ANZCTR): ACTRN12611001251910.

\section{Author details}

'Sax Institute, Haymarket, Australia. ${ }^{2}$ School of Public Health, University of Sydney, Camperdown, Australia. ${ }^{3}$ Cancer Research Division, Cancer Council NSW, Sydney, Australia. ${ }^{4}$ Griffith Health Institute, Griffith University, Gold 
Coast, QLD, Australia. ${ }^{5}$ Department of Radiation Oncology, Royal North Shore Hospital, Sydney, Australia. ${ }^{6}$ Northern Clinical School, University of Sydney, Camperdown, Australia. ${ }^{7}$ NSW Agency for Clinical Innovation, Sydney, Australia. ${ }^{8}$ Westmead Private Hospital, Westmead, Australia. ${ }^{9}$ Westmead Clinical School, University of Sydney, Camperdown, Australia. ${ }^{10}$ Prostate Cancer Foundation of Australia, Melbourne, Australia. ${ }^{11}$ School of Public Health and Community Medicine, University of New South Wales, Sydney, Australia. ${ }^{12}$ School of Medicine and Public Health, University of Newcastle, Callaghan, NSW, Australia.

Received: 18 April 2014 Accepted: 22 May 2014

Published: 29 May 2014

\section{References}

1. Grol R, Grimshaw J: From best evidence to best practice: effective implementation of change in patients' care. Lancet 2003, 362(9391):1225-1230.

2. Haines A, Kuruvilla S, Borchert M: Bridging the implementation gap between knowledge and action for health. Bull World Health Organ 2004 82(10):724-731.

3. Grol R: Successes and failures in the implementation of evidence-based guidelines for clinical practice. Medical Care 2001, 39:||-46-II-54.

4. Buchan $\mathrm{H}$, Sewell JR, Sweet M: Adopting Best Evidence in Practice: Translating evidence into practice. Med J Australia 2004, 180(Suppl 6):s43-s44.

5. Westfall JM, Mold J, Fagnan L: Practice-Based Research-"Blue Highways" on the NIH Roadmap. J Am Med Assoc 2007, 180(Suppl 6):s43-s44.

6. Dougherty D, Conway P: The " 3 T's" road map to transform US health care. JAMA 2008, 299(19):2319-2321.

7. Sung N, Crowley WJ, Genel M, Salber P, Sandy L, Sherwood L, Johnson S, Catanese V, Tilson H, Getz K, Larson EL, Scheinberg D, Reece EA, Slavkin H, Dobs A, Grebb J, Martinez RA, Korn A, Rimoin D: Central challenges facing the national clinical research enterprise. JAMA 2003, 289:1278-1287.

8. Rubenstein L, Pugh J: Strategies for Promoting Organizational and Practice Change by Advancing Implementation Research. J Gen Intern Med 2006, 21(Suppl 2):S58-S64.

9. Hakkennes S, Dodd K: Guideline implementation in allied health professions: a systematic review of the literature. Qual Saf Health Care 2008, 17(4):296-300

10. Baker R, Camosso-Stefinovic J, Gillies C, Shaw EJ, Cheater F, Flottorp S, Robertson N: Tailored interventions to overcome identified barriers to change: effects on professional practice and health care outcomes. Cochrane Database Syst Rev 2010, (Issue 3):Art. No.: CD005470

11. Francke AL, Smit MC, de Veer AJ, Mistiaen P: Factors influencing the implementation of clinical guidelines for health care professionals: a systematic meta-review. BMC Med Inform Decis Mak 2008, 8(1):38.

12. Cabana MD, Rand CS, Powe NR, Wu AW, Wilson MH, Abboud PA, Rubin HR: Why don't physicians follow clinical practice guidelines? A framework for improvement. JAMA 1999, 282(15):1458-1465.

13. Grol $\mathrm{R}$, Wensing $\mathrm{M}$ : What drives change? Barriers to and incentives for achieving evidence-based practice. Med J Aust 2004, 180(6 Suppl):S57-S60

14. Grol R: Has guideline development gone astray? Yes. Br Med J 2010, 340:c306.

15. Greenhalgh T, Robert G, Macfarlane F, Bate P, Kyriakidou O: Diffusion of Innovations in Service Organizations: Systematic Review and Recommendations. Milbank Q 2004, 82(4):581-629.

16. Davies $P$, Walker AE, Grimshaw JM: A systematic review of the use of theory in the design of guideline dissemination and implementation strategies and interpretation of the results of rigorous evaluations. Implementation Sci 2010, 5(1):14

17. Goodwin N, 6 P, Peck E, Freeman T, Posaner R: Managing across diverse networks of care: lessons from other sectors In Report for the National Co-ordinating Centre for NHS Service Delivery and Organisation R \& D (NCCSDO); 2004 [http://www.nets.nihr.ac.uk/ data/assets/pdf file/0006/ 64455/FR-08-1218-039.pdf]

18. Stewart GJ, Dwyer JM, Goulston KJ: The Greater Metropolitan Clinical Taskforce: an Australian model for clinician governance. Med J Aust 2006, 184(12):597-598.

19. Laliberte L, Fennell ML, Papandonatos G: The relationship of membership in research networks to compliance with treatment guidelines for earlystage breast cancer. Med Care 2005, 43(5):471-479.
20. Ray-Coquard I, Philip T, De Laroche G, Froger X, Suchaud JP, Voloch A, Mathieu-Daudé H, Fervers B, Farsi F, Browman GP: A controlled 'before-after' study: impact of a clinical guidelines programme and regional cancer network organization on medical practice. Br J Cancer 2002, 86(3):313-321.

21. ACIM (Australian Cancer Incidence and Mortality) Books. In [http://www aihw.gov.au/acim-books/

22. Catalona WJ, Smith DS, Ratliff TL, Basler JW: Detection of organ-confined prostate cancer is increased through prostate-specific antigen-based screening. JAMA 1993, 270(8):948-954.

23. Partin AW, Kattan MW, Subong ENP, Walsh PC, Wojno KJ, Oesterling JE, Scardino PT, Pearson JD: Combination of prostate-specific antigen, clinical stage, and Gleason score to predict pathological stage of localized prostate cancer [Erratum in JAMA 1997 Jul 9;278(2):118]. JAMA 1997, 277(18):1445-1451.

24. Partin AW, Pound CR, Clemens JQ, Epstein Jl, Walsh PC: Serum PSA after anatomic radical prostatectomy. The Johns Hopkins experience after 10 years. Urol Clin North Am 1993, 20(4):713-725.

25. Pound CR, Partin AW, Epstein Jl, Walsh PC: Prostate-specific antigen after anatomic radical retropubic prostatectomy: patterns of recurrence and cancer control. Urol Clin North Am 1997, 24(2):395-406.

26. National Health Priority Action Council (NHPAC): National Service Improvement Framework for Cancer. In Canberra: Australian Government Department of Health and Ageing; 2006.

27. Bolla M, van Poppel H, Collette L, van Cangh P, Vekemans K, Da Pozzo L, de Reijke TM, Verbaeys A, Bosset J-F, van Velthoven R, Marechal J-M, Scalliet P, Haustermans K, Pierart M, for the European Organization for Research and Treatment of Cancer: Postoperative radiotherapy after radical prostatectomy: a randomised controlled trial (EORTC trial 22911). Lancet 2005, 366(9485):572-578.

28. Swanson GP, Thompson IM, Tangen C, Miller G, Lucia MS, Troyer DA, Paradelo J, Chin JL, Messing EM, Canby-Higano E, Forman J, Crawford DE: Phase III Randomized Study of Adjuvant Radiation Therapy versus Observation in Patients with Pathologic T3 Prostate Cancer (SWOG 8794). Int I Radiat Oncol Biol Phys 2005, 63(1):S1.

29. Wiegel T, Bottke D, Steiner U, Siegmann A, Golz R, Storkel S, Willich N, Semjonow A, Souchon R, Stockle M, Rübe C, Weissbach L, Althaus $P$, Rebmann U, Kälble T, Feldmann HJ, Wirth M, Hinke A, Hinkelbein W, Miller K: Phase III postoperative adjuvant radiotherapy after radical prostatectomy compared with radical prostatectomy alone in $\mathrm{pT3}$ prostate cancer with postoperative undetectable prostate-specific antigen: ARO 96-02/AUO AP 09/95. J Clin Oncol 2009, 27(18):2924-2930.

30. Thompson IM, Tangen CM, Paradelo J, Lucia MS, Miller G, Troyer D, Messing E, Forman J, Chin J, Swanson G, Canby-Hagino E, Crawford ED: Adjuvant radiotherapy for pathological T3NOMO prostate cancer significantly reduces risk of metastases and improves survival: long-term followup of a randomized clinical trial. J Urol 2009, 181(3):956-962.

31. Australian Cancer Network Management of Metastatic Prostate Cancer Working Party: Clinical Practice Guidelines for the Management of Locally Advanced and Metastatic Prostate Cancer. In Sydney: Cancer Council Australia and Australian Cancer Network; 2010.

32. Adjuvant and Salvage Radiotherapy After Prostatectomy: ASTRO/AUA Guideline. In [http://www.auanet.org/education/guidelines/radiation-afterprostatectomy.cfm]

33. Smith DP, King MT, Egger S, Berry MP, Stricker PD, Cozzi P, Ward J, O'Connell $\mathrm{DL}$, Armstrong BK: Quality of life three years after diagnosis of localised prostate cancer: population based cohort study. BMJ 2009, 339:12.

34. Bolton D, Severi G, Millar JL, Kelsall H, Davidson A-J, Smith C, Bagnato M, Pedersen J, Giles G, Syme R: A whole of population-based series of radical prostatectomy in Victoria, 1995 to 2000. Aust N Z J Public Health 2009, 33(6):527-533.

35. Evans S, Millar J, Davis I, Murphy D, Bolton D, Giles G, Frydenberg M, Andrianopoulos N, Wood J, Frauman A, Costello AJ, McNeil JJ: Patterns of care for men diagnosed with prostate cancer in Victoria from 2008 to 2011. Med J Aust 2013, 198(10):540-545.

36. Hoffman K, Nguyen P, Chen M, Chen R, Choueiri T, Hu J, Kuban D, D'Amico A: Recommendations for post-prostatectomy radiation therapy in the United States before and after the presentation of randomized trials. J Urol 2011, 185(1):116-120

37. Flanagan ME, Ramanujam R, Doebbeling BN: The effect of provider-and workflow-focused strategies for guideline implementation on provider acceptance. Implementation Sci 2009, 4(1):71. 
38. Grimshaw JM, Shirran L, Thomas R, Mowatt G, Fraser C, Bero L, Grilli R, Harvey E, Oxman A, O'Brien MA: Changing provider behavior: an overview of systematic reviews of interventions. Med Care 2001, 39(8 Suppl 2):II-2-||-45.

39. Robertson N, Baker $\mathrm{R}$, Hearnshaw $\mathrm{H}$ : Changing the clinical behavior of doctors: a psychological framework. Qual Health Care 1996, 5(1):51-54.

40. Grol R: Implementing guidelines in general practice care. Qual Health Care 1992, 1(3):184-191.

41. Oxman A, Thomson M, Davis D, Haynes R: No magic bullets: a systematic review of 102 trials of interventions to improve professional practice. Can Med Assoc J 1995, 153(10):1423-1431.

42. Flodgren G, Parmelli E, Doumit G, Gattellari M, O'Brien MA, Grimshaw J, Eccles MP: Local opinion leaders: effects on professional practice and health care outcomes. Cochrane Database Syst Rev 2011, (Issue 8):Art. No.: CD000125.

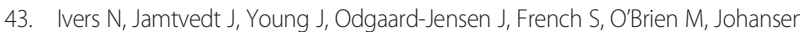
M, Grimshaw J, Oxman A: Audit and Feedback: effects on professional practice and healthcare outcomes (Review). Cochrane Database Syst Rev 2012, (Issue 6):Art.No.: CD000259.

44. Giguere A, Legare F, Grimshaw J, Turcotte S, Flander M, Grudniewicz A, Makosso-Kallyth S, Wolf F, Famer A, Gagnon M: Printed educational materials: effects on professional practice and healthcare outcomes (Review) Cochrane Database Syst Rev 2013, (Issue 4):Art.No.: CD004398.

45. Forstetlund L, Bjorndal A, Rshidian A, Jamtvedt G, O'Brien M, Wolf F, Davis DA, Odgaard-Jensen J, Oxman A: Continuing education meetings and workshops: effects on professional practice and health care outcomes (Review). Cochrane Database Syst Rev 2012, (Issue 11):Art.No.: CD003030.

46. Grimshaw J, Eccles M, Lavis J, Hill S, Squires J: Knowledge translation of research findings. Implementation Sci 2012, 7:50.

47. Grimshaw JM, Thomas RE, MacLennan G, Fraser C, Ramsay CR, Vale L, Whitty P, Eccles MP, Matowe L, Shirran L, Wensing M, Dijkstra R, Donaldson C: Effectiveness and efficiency of guideline dissemination and implementation strategies. Health Technol Assess 2004, 8(6):1-72. iii-iv.

48. Michie S, Johnston M, Abraham C, Lawton R, Parker D, Walker A, Group botPT: Making psychological theory useful for implementing evidence based practice: a consensus approach. Qual Saf Health Care 2005, 14:26-33.

49. Dulko D, Hertz E, Julien J, Beck S, Mooney K: Implementation of cancer pain guidelines by acute care nurse practitioners using an audit and feedback strategy. J Am Acad Nurse Pract 2010, 22(1):45-55.

50. Prior M, Guerin M, Grimmer-Somers K: The effectiveness of clinical guideline implementation strategies-a synthesis of systematic review findings. J Eval Clin Pract 2008, 14(5):888-897.

51. Boaz A, Baeza J, Fraser A, (EIS) EISCG: Effective implementation of research into practice: an overview of systematic reviews of the health literature. BMC Res Notes 2011, 4:212.

52. Davis DA, Taylor-Vaisey A: Translating guidelines into practice: a systematic review of theoretic concepts, practical experience and research evidence in the adoption of clinical practice guidelines. Can Med Assoc J 1997, 157(4):408-416.

53. Hysong SJ, Best RG, Pugh JA: Clinical practice guideline implementation strategy patterns in Veterans Affairs primary care clinics. Health Serv Res 2007, 42(1 Pt 1):84-103.

54. Lankshear S, Brierley JD, Imrie K, Yurcan M: Changing physician practice: an evaluation of knowledge transfer strategies to enhance physician documentation of cancer stage. Healthcare quarterly (Toronto, Ont) 2010, 13(1):84-92.

55. Boxer M, Forstner D, Kneebone A, Delaney G, Koh E-S, Fuller M, Kaadan N: Impact of a real-time peer review audit on patient management in a radiation oncology department. J Med Imaging Radiat Oncol 2009, 53(4):405-411.

56. Blayney DW, McNiff K, Hanauer D, Miela G, Markstrom D, Neuss M Implementation of the Quality Oncology Practice Initiative at a university comprehensive cancer center. J Clin Oncol 2009, 27(23):3802-3807.

57. Brouwers MC, Garcia K, Makarski J, Daraz L, EE CICPRTE: The landscape of knowledge translation interventions in cancer control: What do we know and where to next? A review of systematic reviews. Implementation Sci 2011, 6:130

58. Davey P, Brown E, Fenelon L, Finch R, Gould I, Hartman G, Holmes A, Ramsay C, Taylor E, Wilcox M, Wiffen PJ: Interventions to improve antibiotic prescribing practices for hospital inpatients. Cochrane Database Syst Rev 2005, (Issue 4):Art. No.: CD003543.
59. Arnold SR, Straus SE: Interventions to improve antibiotic prescribing practices in ambulatory care. Cochrane Database Syst Rev 2005, (Issue 4): Art. No.: CD003539.

60. Chaillet N, Dubé E, Dugas M, Audibert F, Tourigny C, Fraser WD, Dumont A Evidence-based strategies for implementing guidelines in obstetrics: a systematic review. Obstet Gynecol 2006, 108(5):1234-1245.

61. Chaillet N, Dumont A: Evidence-based strategies for reducing cesarean section rates: a meta-analysis. Birth 2007, 34(1):53-64.

62. Davis D, Evans M, Jadad A, Perrier L, Rath D, Ryan D, Sibbald G, Straus S, Rappolt S, Wowk M, Zwarenstein M: The case for knowledge translation: shortening the journey from evidence to effect. BMJ 2003, 327(7405):33-35

63. Green LW, Kreuter MW: Health Promotion Planning: An Educational and Environmental Approach. 2nd edition. Mountain View, California: Mayfield Publishing; 1991

64. Sales A, Smith J, Curran G, Kochevar L: Models, strategies, and tools. Theory in implementing evidence-based findings into health care practice. J Gen Intern Med 2006, 21(Suppl 2):S43-S49.

65. Greenhalgh T, Robert G, Bate P, Kyriakidou O, Macfarlane F, Peacock R: How to spread good ideas: A systematic review of the literature on diffusion, dissemination and sustainability of innovations in health service delivery and organisation. In Report for the National Co-ordinating Centre for NHS Service Delivery and Organisation R \& D (NCCSDO); 2004 [http://www.nets. nihr.ac.uk/_data/assets/pdf_file/0017/64340/FR-08-1201-038.pdf]

66. Michie S, van Stralen MM, West R: The behaviour change wheel: A new method for characterising and designing behaviour change interventions. Implementation Sci 2011, 6:42

67. Brown CA, Lilford RJ: The stepped wedge trial design: a systematic review. BMC Med Res Methodol 2006, 6:54.

68. Zwarenstein M, Treweek S, Gagnier JJ, Altman DG, Tunis S, Haynes B, Oxman AD, Moher D, group C, Pragmatic Trials in Healthcare g: Improving the reporting of pragmatic trials: an extension of the CONSORT statement. BMJ 2008, 14:2390.

69. Campbell M, Piaggio G, Elbourne D, Altman D, Group. ftC: Consort 2010 statement: extension to cluster randomised trials. Br Med J 2012, 345:e5661.

70. ARIA. In [http://www.adelaide.edu.au/apmrc/research/projects/category/aria.html]

71. Australian Bureau of Statistics: Information paper: an introduction to socioeconomic indexes for areas (SEIFA). Canberra: ABS; 2008.

72. Young JM, Leong DC, Armstrong K, O'Connell D, Armstrong BK, Spigelman AD, Ackland S, Chapuis P, Kneebone AB, Solomon MJ: Concordance with national guidelines for colorectal cancer care in New South Wales: a population-based patterns of care study. MJA 2007, 186:292-295.

73. Vinod SK, O'Connell DL, Simonella L, Delaney GP, Boyer M, Peters M, Miller D, Supramaniam R, McCawley L, Armstrong B: Gaps in optimal care for lung cancer. J Thorac Oncol 2008, 3(8):871-879.

74. Vinod SK, Simonella L, Goldsbury D, Delaney GP, Armstrong B, O'Connell DL: Underutilization of radiotherapy for lung cancer in New South Wales. Aust Cancer 2010, 116(3):686-694

75. Sladek R, Bond M, Huynh L, Chew D, Phillips P: Thinking styles and doctors' knowledge and behaviours relating to acute coronary syndromes guidelines. Implementation Sci 2008, 3:23.

76. Hakkennes S, Green S: Measures for assessing practice change in medical practitioners. Implementation Sci 2006, 1:29

77. Haines M, Brown B, Craig J, D'Este C, Elliott E, Klineberg E, McInnes E, Middleton S, Paul C, Redman S, Yano EM, on behalf of the Clinical Networks Research Group: Determinants of successful clinical networks: the conceptual framework and study protocol. Implementation Sci 2012, 7:16

doi:10.1186/1748-5908-9-64

Cite this article as: Brown et al:: Clinician-led improvement in cancer care (CLICC) - testing a multifaceted implementation strategy to increase evidence-based prostate cancer care: phased randomised controlled trial study protocol. Implementation Science 2014 9:64. 